\title{
Erratum
}

\section{Shetti, a simple tool to parse, manipulate and search large dataset of sequences}

Haitham Sobhy

MGen, 2015, 1. DOI: 10.1099/mgen.0.000035

The Publisher apologizes for the following error in the manuscript, 'Mati parses UniProt Knowledgebase and NCBI GenBank flat files and visualizes them as a table' 10.1099/mgen.0.000041 which should be 'Shetti parses UniProt Knowledgebase and NCBI GenBank flat files and visualizes them as a table'. 